\title{
Branching and percolation in lecithin wormlike micelles studied by dielectric spectroscopy
}

\author{
P. A. Cirkel, J. P. M. van der Ploeg, and G. J. M. Koper \\ Leiden Institute of Chemistry, Leiden University, Gorlaeus Laboratories, P.O. Box 9502, 2300 RA Leiden, The Netherlands
}

(Received 17 October 1997)

\begin{abstract}
Lecithin water-in-oil microemulsions have been shown to form long polymerlike micelles. Dielectric spectra of this system are characterized by two dispersions. The high frequency dispersion, related to the head-group rotation of the lecithin molecule, displays a different dependence on water addition in the same two regimes that show up differently in the dynamics measured with several other techniques. The low frequency dispersion is due to a polymeric Rouse/Zimm type mode, which above a certain concentration starts to decrease and shows the characteristics of percolation. In the high water regime the decrease of the relaxation time is accompanied by an increase in conductivity, whereas in the low water regime the conductivity decreases. These data are interpreted in terms of concentration induced percolation and water induced coalescence into a connected network. [S1063-651X(98)05606-2]
\end{abstract}

PACS number(s): 82.70.Kj, 61.25.Hq, 77.22.- d, 87.15.Da

\section{INTRODUCTION}

Among the various structures that form in surfactant systems, long flexible micelles of cylindrical shape have during recent years received quite some attention [1] because their physical properties, structure, and thermodynamic behavior are reminiscent of living polymer solutions. For example, an overlap volume fraction $\phi^{*}$ has been defined above which these micelles are believed to entangle so that their equilibrium properties can be described by scaling theory [2]. However, for wormlike micelles $\phi^{*}$ is not as well defined as for conventional polymer systems due to the broad micellar length distribution. Nevertheless, at sufficiently high volume fractions, experiments show perfect agreement with scaling theory for polymer solutions. Although there are quite a few surfactants that form these wormlike micelles in aqueous systems, lecithin with trace amounts of water is one of the few systems that forms reverse wormlike micelles in organic solvents. In this system the agreement with scaling theory has been demonstrated by static and dynamic light scattering [3]. More controversial are the dynamic properties of wormlike micelles. A model that describes the dynamic behavior of these so-called equilibrium polymers, i.e., linear macromolecules that can break and recombine (reversible scission), has been developed by Cates [4]. Many experimental observations on wormlike micelles are in agreement with this model but there appear to be discrepancies as well. It is remarkable, for instance, that as a function of water content in lecithin wormlike micelles [5], there initially is an enormous increase in the zero shear viscosity, in agreement with the micellar growth observed by other techniques, but that above a certain water content the viscosity falls dramatically again. An explanation is that the micelles are fusing to form a connected network [6]. This would reduce the viscosity because the branch points are not chemical connections and can slide along the branches [7] or act as a release of entanglements via a transient branch point [8]. In aqueous systems the existence of branched micelles has been demonstrated by cryotransmission electron microscopy $[9,10,11]$. Unfortunately cryo-TEM experiments in organic solvents such as iso-octane are much more difficult to perform [12].
In this paper we investigate possible branching in the system of lecithin with trace amounts of water in iso-octane, using dielectric spectroscopy. This system is suitable for a study by dielectric spectroscopy since it contains anisotropic structures with a high dielectric permittivity dispersed in a solvent with a low permittivity. We recently studied this system by electro-optic birefringence for the same reason [13]. An advantage of dielectric spectroscopy, above other techniques that probe the dynamics of a system, is that it also measures the conductivity. The formation of a connected network containing water in oil structures can be expected to have an impact on the electric conductivity, since it would resemble the situation of percolation in, for instance, dropletphase water-in-oil microemulsions [14]. It will be postulated that the coils or networks of wormlike micelles aggregate or entangle upon increase of the concentration to ultimately form a percolated network at a water-content-dependent critical value. Above a certain content of water the micelles not only aggregate or entangle but also form junctions so that the percolating network becomes electrically conductive. It will be shown in this paper that such a percolation model is able to describe the anomalies in the dynamics of this system reported both here and in the literature.

\section{EXPERIMENTAL SECTION}

The soybean lecithin used in these experiments was obtained from Lucas Meyer (Hamburg, Germany), type Epicuron 200. It was used without further purification, which consequently means that it is a certain mixture of surfactants of different chain length and degree of saturation [15]. Isooctane was of analytical grade and purchased from Merck (Darmstadt, Germany). We identify the samples by their surfactant volume fraction $\phi$ (taking for the density of lecithin $\rho=1.056 \mathrm{~kg} / \mathrm{l})$, which because of the negligible influence of the added water is also equal to the volume fraction of micelles, and $w_{0}$, the molar ratio of added water to surfactant. The abundance of water already present in the lecithin was determined to be approximately $0.7 \mathrm{~mol}$ per mol of lecithin (by IR spectroscopy and Karl-Fisher titration). This value should be added to the $w_{0}$ values as used here to obtain the actual water to surfactant ratio in the samples. 
Impedance spectra were recorded with an impedance analyzer, HP4194A (Hewlett-Packard, San Diego, CA), in the high-frequency range $(100 \mathrm{~Hz}$ to $100 \mathrm{MHz})$. In the lowfrequency range (from $1 \mathrm{~Hz}$ to $10 \mathrm{kHz}$ ) a lock-in amplifier, SR510 (Stanford Research Systems, Sunnyvale, CA.), was used in combination with an external signal generator, HM3180 (Hameg GmbH, Frankfurt am Main, Germany). The analyzers were connected to cylindrical cells with various electrode separations. The measured impedance was interpreted in terms of a parallel circuit of a capacitance and a conductance, yielding both the dielectric permittivity and the conductivity versus frequency. The real parts of two different functions, which are both generalizations of the Debye function [16] describing the spectrum of a first-order relaxation process, were used to fit to the measured dielectric permittivity spectra. These are

(i) the Cole-Cole function

$$
\varepsilon=\varepsilon_{\infty}+\frac{\varepsilon_{s}-\varepsilon_{\infty}}{1+(i \omega \tau)^{\beta}}
$$

and (ii) the Cole-Davidson function

$$
\varepsilon=\varepsilon_{\infty}+\frac{\varepsilon_{s}-\varepsilon_{\infty}}{(1+i \omega \tau)^{\beta}}
$$

with $\omega$ the circular frequency, $\tau$ the characteristic relaxation time, and $\varepsilon_{s}$ and $\varepsilon_{\infty}$ the permittivity at $\omega \tau \ll 1$ and $\omega \tau \gg 1$, respectively. In general, if a distribution of relaxation times is smooth and not too broad, the Cole-Cole function can be used for an almost symmetrical distribution whereas the Cole-Davidson function can be used in the case of a highly asymmetrical distribution of relaxation times. In both functions, $\beta$ describes the broadness of the distribution. The higher $\beta$ the smaller the distribution of relaxation times and with $\beta=1$ the Debye function is recovered describing one single relaxation time. We also analyzed the spectra by an inverse Laplace transformation routine. This did, however, not give very satisfactory results since the variation in experimental error resulted in an inconsistent way in which the so-called smoothness parameter had to be used in order to obtain a regular variation of the relaxation time with volume fraction $\phi$.

\section{RESULTS}

Two typical relaxation spectra are shown in Fig. 1(a). In the measured dielectric spectra we distinguish two dispersions and a decrease of the permittivity at very low frequencies. This decrease strongly depends on electrode spacing. Therefore it has to be due to the diffusion of charged particles over a distance comparable to this spacing, the socalled electrode polarization; possible slower relaxation processes will not be detected because of this electrode polarization. This effect could be separated from the rest of the spectrum by choosing a sufficiently large electrode spacing as we proposed in Ref. [17].

At low frequencies there is a dispersion that becomes asymmetrical at high surfactant volume fractions and high values of $w_{0}$. The volume fraction dependence of the characteristic relaxation time, $\tau_{1}$, for this dispersion is shown in Fig. 2(a). The closed symbols in this figure correspond to
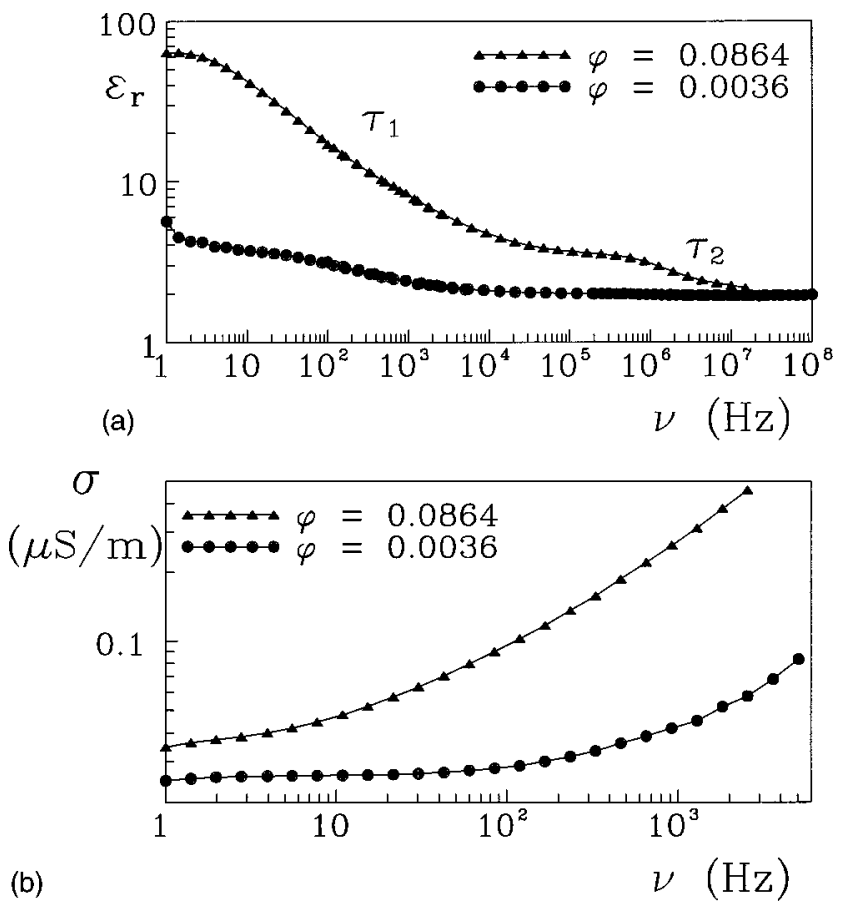

FIG. 1. (a) Dielectric spectra for $w_{0}=2.5$ at two volume fractions. In the spectrum for $\phi=0.0864$ the dispersion related to $\tau_{1}$ is asymmetrical, and is more sharply curved at low frequencies. (b) Conductivity spectra for $w_{0}=2.5$ at two volume fractions.

relaxation times obtained by fitting the asymmetrical ColeDavidson function, whereas the others were obtained with the Cole-Cole function. The qualitative difference in these two regimes is shown in the spectra of Fig. 1(a). For $w_{0}$ $<2$ the characteristic relaxation time $\tau_{1}$ is almost constant at low volume fractions and increases with approximately volume fraction squared at higher volume fractions. For $w_{0}$ $>2$ there is a scaling regime at low volume fractions, see Table I. Above a critical volume fraction $\phi_{c}$, the dispersion becomes asymmetrical and the relaxation time decreases towards a lower level. The increment in permittivity of this dispersion scales with the volume fraction as well (see Table I) and levels off above $\phi_{c}$ see Fig. 2(b). The distribution of relaxation times in this dispersion is rather broad, with width parameter $\beta \approx 0.5$. In the regime of constant $\tau_{1}, \beta$ appears to be constant and subsequently the distribution gets broader with higher volume fractions, until $\phi_{c}$, where it becomes narrower again, see Fig. 2(c). The volume fraction $\phi_{c}$ decreases with $w_{0}$, the molar water-to-surfactant ratio.

At high frequencies there is a dispersion that is symmetrical and can hence be fit to the Cole-Cole function. The characteristic relaxation time, $\tau_{2}$, for this dispersion does not depend on volume fraction but decreases with $w_{0}$, as is demonstrated in Fig. 3(a). There appear to be two regimes, in both of which the relaxation time is a negative exponential of $w_{0}$ with a faster decay from 0.5 to 2 than from 2 to 4.5 . The increment in permittivity corresponding to this dispersion depends linearly on volume fraction: see Fig. 3(b). The values for the width parameter $\beta$ are always rather high, but clearly different from 1 with a minimum for $w_{0}=2$ [Fig. 3(c)].

The conductivity spectra display a plateau at low frequencies, see Fig. 1(b). The value of the conductivity at this plateau is shown in Fig. 4 as a function of $w_{0}$ and $\phi$. Again the 

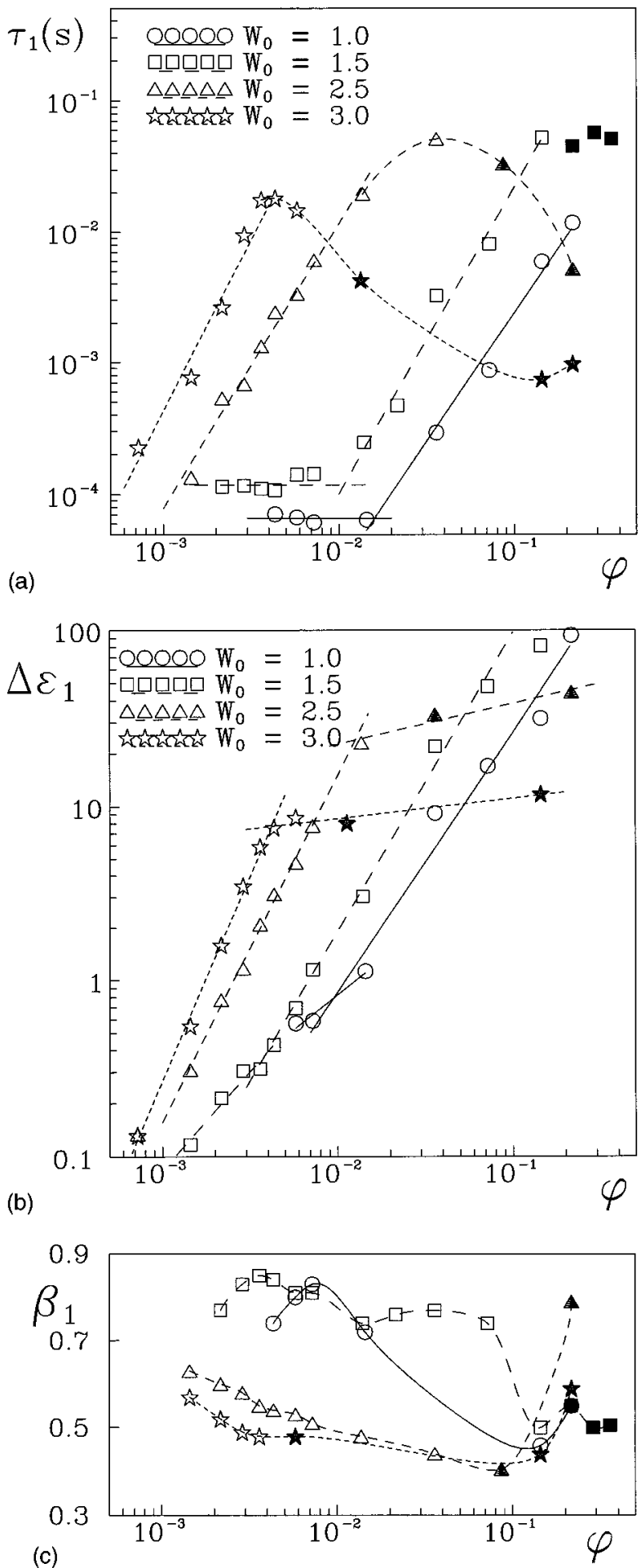

FIG. 2. (a) The relaxation time related to the low frequency dispersion. The straight lines indicate power law fits to the data (exponents displayed in Table I). The bended lines are guides to the eye. Open symbols are obtained by fitting the spectra to the Cole/ Cole function whereas closed were obtained by the Cole/Davidson function (see text). (b) The dielectric increment related to the low frequency dispersion. The lines indicate power law fits to the data (exponents displayed in Table I). (c) The broadness parameter $\beta$ related to the low frequency dispersion. Lines are guides for the eye. Note that the definition of $\beta$ is different for the filled symbols, where instead of the Cole/Cole function, the Cole/Davidson function was used to fit the data.
TABLE I. Fitted scaling exponents and interpretation of these exponents in terms of polymer theory in combination with micellar growth if $M \propto \varphi^{\alpha}$.

\begin{tabular}{lclllll}
\hline \hline$W_{0}$ & Regime & $\tau_{1}$ & $\alpha_{\tau}$ & $\Delta \epsilon_{1}$ & $\alpha_{\Delta \epsilon}$ & $\sigma$ \\
\hline 0.5 & $\phi<2 \times 10^{-2}$ & & & & & 0.8 \\
1.0 & $\phi<2 \times 10^{-2}$ & 0 & 0 & 0.8 & $\approx 0$ & 1.1 \\
& $\phi>2 \times 10^{-2}$ & 2.0 & 0.5 & 1.5 & Conflict & \\
1.5 & $\phi<1 \times 10^{-2}$ & 0 & 0 & 1.1 & $\approx 0$ & 0.7 \\
& $1 \times 10^{-2}<$ & 2.3 & 0.65 & 1.7 & Conflict & \\
& $\phi<1 \times 10^{-1}$ & & & & & \\
2.5 & $\phi<3 \times 10^{-2}$ & 2.2 & 0.6 & 2.0 & Conflict & 0 \\
3.0 & $\phi<4 \times 10^{-3}$ & 2.6 & 0.8 & 2.3 & Conflict & 0 \\
\hline \hline
\end{tabular}

two $w_{0}$ regimes can be observed. In the low $w_{0}$ regime the conductivity increases linearly with $\phi$ at those volume fractions where $\tau_{1}$ is constant (Table I). At the point where $\tau_{1}$ starts to increase, the conductivity levels off and eventually decreases. In the high $w_{0}$ regime the conductivity is almost constant for low volume fractions and increases more than linearly with the volume fraction above $\phi_{c}$.

We also measured the low frequency dispersion $\left(\tau_{1}\right)$ and the conductivity at $w_{0}=2$. The experimental error in these measurements was significantly larger than for all the other measurements, so we do not show the results here. We will come back to this point in the discussion. For $w_{0}=0.5$ the low frequency dispersion could not be separated from the electrode polarization in a reliable way.

\section{DISCUSSION}

\section{A. The low frequency dispersion}

At low volume fractions the mean relaxation time of the low frequency dispersion is of the order of $10^{-4} \mathrm{~s}$. This corresponds to the rotational diffusion time of a micelle with a hydrodynamic radius such as has been determined in this system by dynamic light scattering [3] and FRAP (fluorescence recovery after photobleaching) [18], i.e., $300 \AA$ A . At higher volume fractions this relaxation time scales with the volume fraction in approximately the same way as was observed by the Kerr effect [19]. It is therefore obvious to compare this dispersion with the rotational correlation time of semi-flexible polymers, which can be described by the leading mode in the model by Rouse and Zimm [20-22]. Higher order modes are in the context of wormlike micelles unimportant since their effect on the distribution of relaxation times is smeared out due to polydispersity. For low volume fractions this relaxation time is constant. For higher volume fractions, upon entering the semidilute regime, polymer theory $[23,24]$ predicts that the lowest order relaxation time picks up a linear $\phi$ dependence with a prefactor that depends on polymer mass, $M$, i.e.,

$$
\tau_{1}=\tau_{1}^{0}\left(1+A M^{3 \nu-1} \phi\right)
$$

with $A$ a constant and $\nu=3 / 5$ the excluded volume exponent for good solvents. To evaluate the full molar mass dependence in this equation, the relation between the molar mass and $\tau_{1}^{0}$ has to be added. From the Zimm model in good 

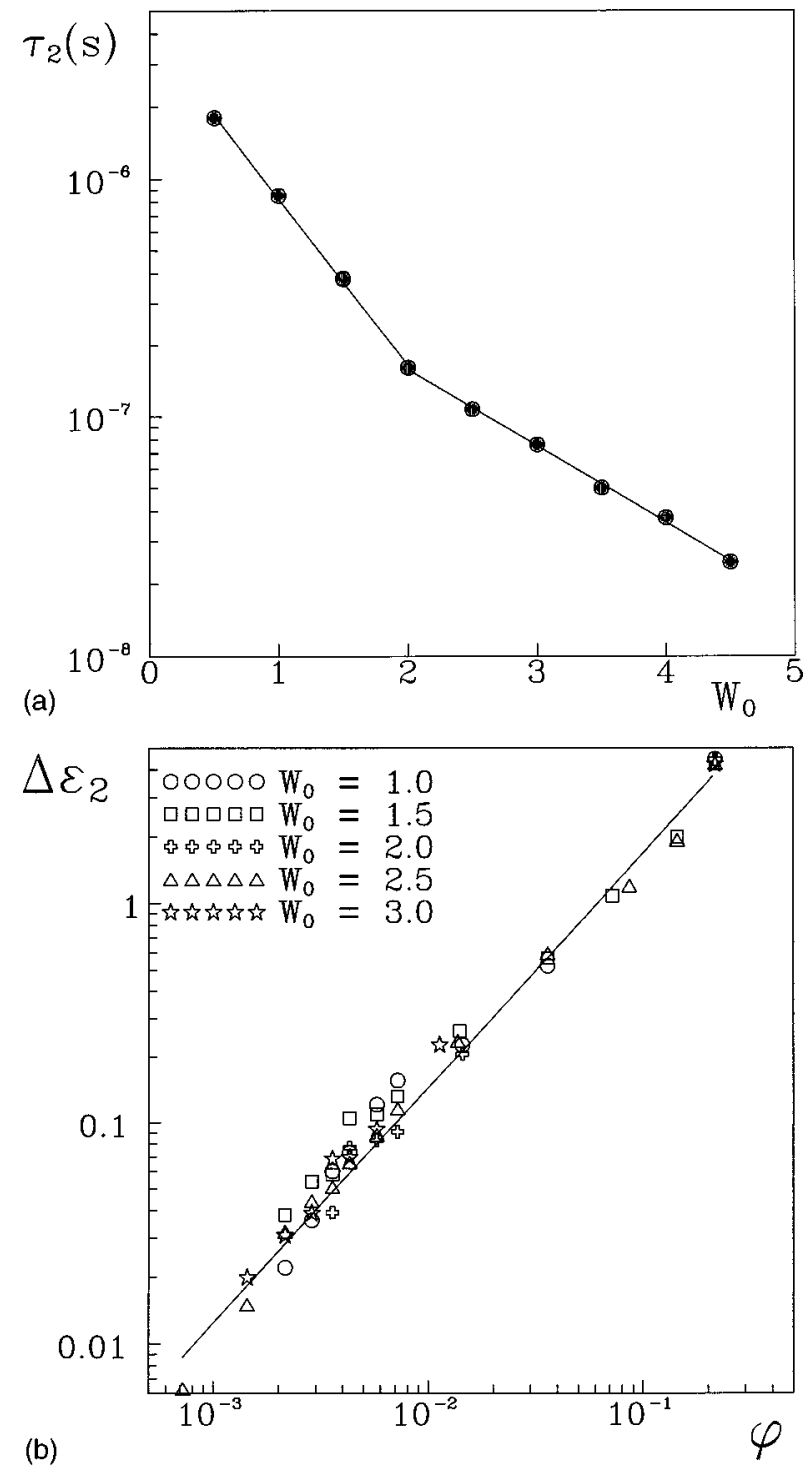

(b)

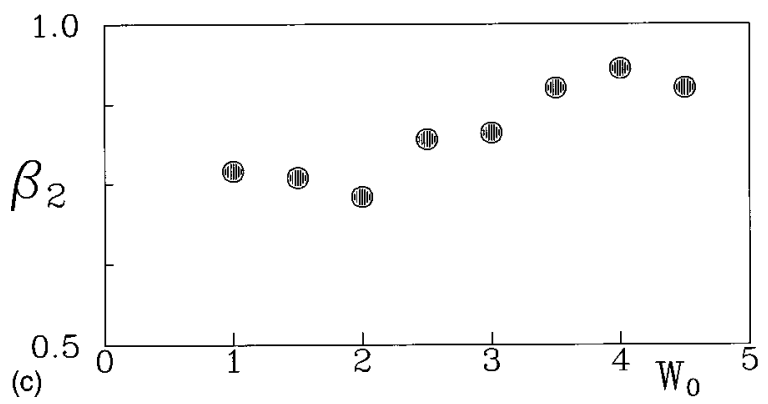

FIG. 3. (a) The relaxation time related to the high frequency dispersion. The line indicates an exponential fit to the data. For $w_{0} \leqslant 3$ the mean value of all different volume fractions was taken, whereas for $w_{0}>3$ the value at $\phi=0.144$ was taken. (b) The dielectric increment related to the high frequency dispersion. The line indicates a linear fit to the data. (c) The broadness parameter $\beta$ related to the high frequency dispersion.

solvents it can be derived that $\tau_{1}^{0} \propto M^{3 \nu}$. The volume fraction dependence of the increment related to the relaxation time can be derived using scaling theory. The relation between the increment, $\Delta \varepsilon$, and the mean squared end-to-end distance, $\left\langle r^{2}\right\rangle$, is given by [20]

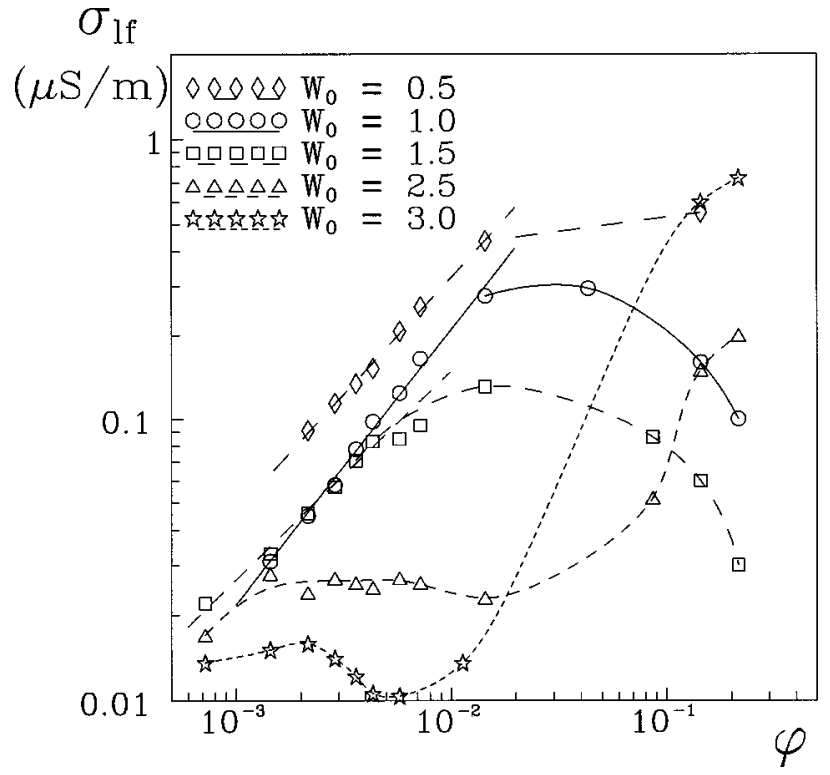

FIG. 4. The conductivity at the low frequency plateau. Straight lines indicate power law fits to the data (exponents displayed in Table 1). Bended lines are guides to the eye.

$$
\Delta \varepsilon=F \frac{\left\langle r^{2}\right\rangle}{M} \phi
$$

with $F$ a factor proportional to the segmental (induced) dipole of the chain. Scaling theory can be used to derive the relation between the mean squared end-to-end distance and the molecular mass. In the dilute regime it is given by [2]

$$
\left\langle r^{2}\right\rangle \propto M^{2 \nu} \rightarrow \Delta \varepsilon \propto M^{2 \nu-1} \phi
$$

and in the semidilute regime by [25]

$$
\left\langle r^{2}\right\rangle \propto M \phi^{(2 \nu-1) /(1-3 \nu)} \rightarrow \Delta \varepsilon \propto \phi^{1+(2 \nu-1) /(1-3 \nu)} .
$$

The volume fraction dependence of the increment should hence change slightly from linear in the dilute regime to $\phi^{3 / 4}$ in the semidilute regime. Note that the increment should become independent of the molecular weight in the semidilute regime. These relations have been thoroughly tested on dielectric spectra from solutions of various conventional polymers [26-29]. In our case, at low volume fractions and $w_{0}$, we observe a constant $\tau_{1}$ and $\Delta \varepsilon$, which depend linearly on the volume fraction (Table I). However, at higher volume fractions or $w_{0}$ both this relaxation time and $\Delta \varepsilon$ increase approximately with the volume fraction squared. Of course, for wormlike micelles the scaling predictions should be modified since the average molar mass is supposed to be a function of the surfactant volume fraction. In general, this function can be written as $M \propto \phi^{\alpha}$. In the mean-field approximation it was calculated that $\alpha=1 / 2[1,30]$. A value of $\alpha$ that is lower than $\frac{1}{2}$ could be accounted for since the number of degrees of freedom that a surfactant loses upon aggregation is unknown [31,32]. Values significantly higher than $\frac{1}{2}$ have not been explained theoretically so far. In our case, however, this approach does not lead to consistent results, since it yields a different value for $\alpha$ depending on both the regime and the specific parameter studied (Table I). A straightforward result can be obtained in the dilute regime where the 
micellar size seems to be concentration independent, i.e., $\alpha$ $\approx 0$, regardless of which parameter is studied. This is confirmed by viscometry and Kerr-effect measurements [33], but contradicted by light-scattering experiments interpreted by renormalization group theory [34], for which one finds $\alpha$ $=1.2$. It is also consistent with the observation that the low frequency conductivity in this regime increases linearly with the volume fraction. If the mechanism behind the conductivity is the diffusion of micelles, this would mean that these micelles are constant in size and only interact weakly. Regimes with low values for $\alpha$ have been observed in other surfactant systems, by using the Kerr effect [35] and from light-scattering experiments in very dilute solutions [36].

In the semidilute regime the determination of $\alpha$ appears to be ambiguous. The relaxation time would indicate that $\alpha$ $\approx 0.5$ whereas the interpretation of the increment is in conflict with polymer theory, which predicts scaling with an exponent of $\frac{3}{4}$ on $\phi$ and an independence with respect to the molecular weight. The scaling relations used to obtain these results could be modified slightly by the semiflexibility of the chain or by interactions but these factors alone seem to be insufficient to give a consistent result, particularly since experimental results on polymers have been found to be quite universal. Moreover, although it is expected that $\alpha$ is slightly higher in the semidilute than in the dilute regime [37], a difference in $\alpha$ of more than 0.5 as observed here seems to be too large as well.

Beyond the semidilute regime scaling is followed by a maximum in the relaxation time at rather small volume fractions. For $w_{0}=3$ this maximum already occurs at $\phi_{c}$ $\approx 0.4 \%$. At the same volume fraction the distribution of relaxation times becomes asymmetrical and the corresponding dielectric increment levels off as a function of volume fraction. These observations indicate the presence of a cutoff in the relaxation time distribution for long relaxation times. One could argue that this is due to the polymers getting entangled $\left(\phi^{*} \approx 0.5 \%\right.$; static light scattering [3]). However both experiment as well as theory for polymers show that for dynamical properties the volume fraction at which this occurs for $w_{0}=3$ is far too low to pose a real restriction for the chains to relax via modes corresponding to length scales longer than the transient network correlation length. Even for much larger volume fractions one expects on the basis of polymer theory that the relaxation time only shifts to moderately larger values as is calculated by the Muthukumar theory $[23,24]$.

These observations together clearly show that polymer theory together with micellar growth cannot be used to explain all aspects of the low frequency dispersion. One could argue that the dynamic scaling relations are modified by micellar kinetics (i.e., breakup, exchange of surfactants). However, if this would be the case relaxation through rotational diffusion would be almost single exponential (fastest and slowest relaxation time would at most differ by a factor of 3 ) $[38,39]$. For this system, measurements on the complex viscosity show that the terminal relaxation time (i.e., typical time for a combination of breakage and self-diffusion of a micelle) is $0.2 \mathrm{~s}$ [40].

\section{B. The high frequency dispersion}

The high frequency dispersion could in principle have two origins: (i) a local polymerlike motion due to the component of the dipole moment perpendicular to the chain $[21,22]$; (ii) a surfactant motion due to the rotation of the zwitterionic head group [41,42]. It is improbable that in this case the dipole moment would have a component perpendicular to the chain since the cross section is likely to be of circular symmetry. Moreover, a polymerlike dispersion of this type would normally be rather broad and asymmetrical, whereas this dispersion is sharp and symmetrical. It is therefore most likely that this dispersion is due to the rotation of the zwitterionic head group of the lecithin molecules. Such a head group motion was studied in aqueous solutions with dielectric spectroscopy by Kaatze et al. [41,42]. In the lamellar phase they observed a Debye dispersion (i.e., simple first order relaxation process) with a relaxation time of about 25 $\mathrm{ns}$, a value that is very much like the relaxation time we observe for the high frequency dispersion just before phase separation occurs (at $w_{0} \approx 4.8 ; \phi=0.144$ ).

Similar information about the freedom of head groups, which supports the interpretation of this mode in terms of head group rotation, were obtained using FT-IR [43] and NMR [44] spectroscopy. FT-IR spectroscopy experiments show that the position of the peak in the region of the antisymmetric $P=O$ stretch vibration $\left(1220-1260 \mathrm{~cm}^{-1}\right)$ demonstrates roughly the same dependence on $w_{0}$ as this mode. The most striking result from NMR spectroscopy is the almost twofold broadening of the water proton line below $w_{0}=2$ indicating that the water gets more strongly bound [44].

\section{Percolation; the conductivity}

In this section we will combine the information from the low frequency dispersion and the high frequency dispersion with that of the conductivity and the indications for network formation in the literature to show that all of this can be explained using a percolation model. The high frequency dispersion clearly shows that there are two regimes in which the addition of water has a different effect on the head groups. The low frequency dispersion also behaves differently in the same two regimes. Other techniques show different behavior in these two regimes as well. Apart from the NMR linewidth mentioned before, the exponent by which the viscosity scales with the surfactant concentration is anomalously low in the high water regime [45,5]. At $w_{0}=1.5$ this exponent is 3.7 , which is in good agreement with the "equilibrium polymer" model, provided that one takes into account a growth exponent of $\alpha=1 / 2$. The exponent is only 1.9 at $w_{0}=2.5$ and 3 . Abnormal behavior of a dynamic scaling exponent above $w_{0}=2$ has also been observed by measurements of the selfdiffusion coefficient by FRAP (fluorescence recovery after photobleaching) [18]. Similar phenomena have been observed in aqueous systems at high salt content and have been ascribed to the formation of a connected network [46,47]. One would expect that the formation of such a network would have a strong impact on the conductivity of this system, which is oil continuous. For $w_{0}>2$ the conductivity does indeed change its dependence on $\phi$ just at $\phi_{c}$ where $\tau_{1}$ exhibits its maximum. The jump in conductivity is lower than what is observed in percolating water-in-oil droplets, for 
which the conductivity can increase 4 orders of magnitude [14]. This is, however, not surprising since the NMR linewidth shows that at low water content the water is strongly bound [44], so that the conductivity in the branches of the micelles can be expected to be low. This is supported by the observation that the micelles appear to have long-lived dipoles (at the time scale of seconds at least) [13].

Now we could test the hypothesis of a percolation threshold at $\phi_{c}$ for the other observations. The maximum in $\tau_{1}$ seems to fit very nicely with the usual percolation picture [14]: At low volume fractions the rotational correlation time gets slower due to the growth of clusters and due to interactions, which typically follows some kind of scaling law. Then, at the percolation threshold, the biggest cluster spans the whole sample and the mean rotation correlation time starts to decrease because only the smaller clusters are still able to rotate and contribute to the spectrum. A consequence of this mechanism is that the contribution of this relaxation time to, for instance, the dielectric spectrum decreases rather rapidly, whereas in the present case the increment of the low frequency dispersion only levels off. However, one must keep in mind that the percolating structures here are not rigid spheres but wormlike micelles with polymerlike internal degrees of freedom. One specific degree of freedom, which could still be active after percolation is sliding of branches within the network [7]. The fact that the relaxation time spectrum gets highly asymmetrical seems to support this picture: Only the slow parts of the spectrum freeze out upon percolation, whereas the faster internal modes persist.

The percolation threshold decreases with $w_{0}$, which suggests that the number of junctions increases with $w_{0}$. This is supported by the decreased broadness of the maximum and the fact that the increment of the dispersion levels off at different values for different values of $w_{0}$. From the observation of $\tau_{1}$ only, one could also argue that there is no qualitative difference below and above $w_{0}=2$, and the curve is just shifted towards higher volume fractions. This seems to be supported by the fact that at $w_{0}=1.5 \tau_{1}$ also seems to exhibit a maximum at high volume fractions and that at this $\phi_{c}$ the spectrum becomes asymmetrical and the increment levels off. However, the conductivity in this case decreases and it thus seems more likely at this high volume fraction that the importance of entanglements is enough to explain the freezing out of slower modes. The importance of the entanglements could be enhanced if the micelles in these entanglements would stick together and form junctions without the water channels fusing. This is equivalent to what happens in spherical droplet phase microemulsions, where at higher volume fractions the droplets stick together and form aggregates [48]. This could also be the key to explaining the discrepancies with polymer theory for $\tau_{1}$ and $\Delta \varepsilon_{1}$ at lower volume fractions and $w_{0}<2$. In this case, both these parameters scale with anomalously high exponents on the volume fraction. It is striking that these exponents are almost the same as in the case of $w_{0}>2$ below $\phi_{c}$. This is, however, to be expected since by rotational diffusion sticky connections and branch points cannot be distinguished: the same kind of aggregates are set in motion. For other parameters, such as the conductivity or shear viscosity [45], this is not the case and clearly a different scaling is observed.
The percolation picture can be related to the anomalous behavior of the tracer self-diffusion coefficient by FRAP [18]. Below $w_{0}=2$ the expected decrease of the micellar self diffusion coefficient with $w_{0}$ and $\phi$ is observed. For the $\phi$ dependence the power law dependence compares very well with theory. At $w_{0}=2.5$, however, the diffusion coefficient does not vary with the volume fraction (all measured volume fractions $\leqslant \phi_{c}$.) and the diffusion is "accelerated," i.e., $t^{n}$ $=\left\langle r^{2}\right\rangle$ with $n>1$. This could be due to the diffusion of the tracer through a fractal structure like a percolating network. Although in general this would give rise to a deceleration of the diffusion [49], there are some cases in which an acceleration is observed [50]. However, in another aqueous surfactant system, the anomalous diffusion was ascribed to the residing of the probe on smaller and smaller micelles due to abnormal micellar kinetics [51]. At $w_{0}=3$ the diffusion coefficient increases with the volume fraction, first steeply and then less steeply. For this $w_{0}$ the diffusion coefficient was measured at volume fractions just over $\phi_{c}$. Obviously this would fit the percolation picture very well if the tracer diffuses through the cluster. Finally at $w_{0}=4$ the diffusion coefficient is rather high and does not depend on the volume fraction. In this case probably at all volume fractions the network is fully percolated with the branch points at relatively close distances.

At this stage we could try to interpret the observations on the fast mode, $\tau_{2}$, which seems to be related to the surfactant head group rotation. In wormlike micellar solutions a large fraction of the surfactant head groups should be in the cylindrical regions of the micelles and a rather limited fraction constitutes the end caps $(\approx 1 \%)$, so that the head groups in the end caps contribute negligibly to this relaxation time spectrum. This is confirmed by the fact that in toluene, where the micelles seem to be spherical, this relaxation time does not appear at all, probably because the head groups are too much restricted in their rotation. The decrease of the relaxation time in the region from $0.5 \leqslant w_{0} \leqslant 2$ is most probably due to the release of head groups by the swelling of the cylinders. In the region from $2 \leqslant w_{0} \leqslant 4.5$ the dependence of this relaxation time on $w_{0}$ is clearly different, indicating that the mechanism behind the release of head groups is different as well. Apart from swelling, an obvious mechanism for the release of head groups is the formation of branch points. The head groups of the surfactant molecules in the branch points are less restricted in their motion because the surfactant layer is locally less curved towards the water. Addition of water can have two distinct effects: the swelling of the branches of the micelles and the increase of the number of branch points. Both effects have their own $w_{0}$ dependence. The apparent crossover between the two decay modes of the central relaxation time as a function of $w_{0}$, at $w_{0}=2$ (see Fig. 2), could well be explained by the following: for low values of $w_{0}$, one predominantly observes the water content dependence of the head group motion of the surfactants in the branches. For higher values of $w_{0}$ the relative abundance of branch points is sufficiently large so that the contribution of the surfactants in these regions dominates. At $w_{0}=4.5$, the value of this relaxation time is almost the same as in the lamellar phase, which could be a reflection of the fact that if the branch points are close to each other the curvature effectively vanishes. The broadness of the distribution of relaxation times 
also depends on $w_{0}$. This distribution is the broadest just at the crossover $w_{0}=2$. If this dispersion is a combination of two relaxation times, corresponding to surfactants in the cylindrical parts and the branch points, respectively, which are too close to be resolved the distribution would be the broadest at the point were both relaxation times contribute equally. The sharpness of the crossover between the two regimes can be explained by the fact that both the energies of forming an end cap and of forming a branch point are much larger than $k T$. A relative small change of these energies due to the addition of water could change their relative magnitude so that branch points are favored over end caps [6].

\section{Network structure}

Drye and Cates were the first to study theoretically branching in wormlike micellar systems; in their study they used an infinite end-cap energy [6]. Elleuch et al. [52] performed a similar study taking a finite end-cap energy. Both approaches led to two main conclusions. The first concerns the stability of the micellar solution: at high volume fractions the branched micelles build up a network in which branch points and entanglements coexist. This is called an unsaturated network. As $\phi$ is decreased the network gets more saturated, i.e., the ratio of branch points to entanglements gets smaller, until a certain value at which the number of branch points is of the order of one per blob. At lower volume fractions the system expels excess solvent and phase separation follows. The second conclusion concerns the average strand length between branch points, $L_{s}$. This parameter is expected to scale with volume fraction with an exponent, which depends on the number of branches that come together in a branch point. For threefold junctions it takes the form $L_{s} \propto 1 / \sqrt{\phi}$.

It is indeed observed that at high water content the system phase separates upon dilution into a highly viscous solution on the bottom and a phase of almost pure isooctane on top. At room temperature this occurs for $w_{0} \geqslant 3.5$ [5]. However, at $6{ }^{\circ} \mathrm{C}$ we observe phase separation upon dilution at $w_{0}$ $=2.5$. This seems to confirm the qualitatively different behavior below and over $w_{0}=2$ as observed by other techniques. It is consistent with the hypothesis that only at $w_{0}$ $>2$ the system forms junctions that persist upon dilution. The fact that phase separation is temperature dependent indicates that end caps still are still important in the regime $2.5<w_{0}<3.5$. In the case of finite end-cap energy, theory predicts that phase separation occurs in the temperature region where the branch-point energy is much larger than $k T$ [52]. Although below $w_{0}=3.5$ end caps might be important, the conductivity indicates that above $w_{0}=2$ the system percolates to form larger water domains at higher surfactant volume fractions. This situation seems to be reminisent of the networklike structures found by Monte Carlo simulations [53]. In this study the end-cap energy has a finite value and it was shown that, if branching occurs, the occurrence of phase separation critically depends on the ratio of spontaneous curvature and bending rigidity. This study also shows the crucial role of ringlike structures, which could be important in our system as well. It is also interesting to speculate about the structure of the particles in the regime of $2.5<w_{0}<3.5$ at volume fractions below $\phi_{c}$. It seems that these particles are small networks rather than isolated branched micelles, because preliminary NMR experiments show that the water long time self-diffusion is much faster than the surfactant diffusion.

Theoretical predictions for the strand length seem to be in conflict with our data. The relaxation time of the fast mode is concentration independent. If our interpretation is correct this means that the number of branch points per cylindrical length and hence the strand length is concentration independent. This would also explain why, in neutron scattering experiments, the local structure (cross section radius and mass per length) appears to be completely concentration independent [34]. The conflict with theory might not be so surprising considering the complexity of the system. One complicating factor is that branch point energy and end-cap energy may not be meaningful parameters in this case due to the special role played by water. The end-cap energy is increased by the addition of water, whereas the branch point energy seems to be decreased at the same time. If this is true these two energies are related through water partitioning. These factors could have a rather drastic impact on the relation between concentration and strand length. Another factor, which has not yet been taken into account in these theories, is the interaction between branch points via the strand. Given the fact that branch points might actually take up quite a lot of space this might be important as well. This has recently been incorporated in a theory to explain the loop shape of the connected micellar phase (in coexistence with other phases) in the phase diagram for two component systems [54]. In principle the strand length could also be estimated from the concentration dependence of the slow mode. At $w_{0}=3$ this mode seems to become concentration independent. This could be taken as an indication that the strand length is concentration independent as well. One must, however, be cautious since the actual dynamics of a strand are governed by complicated interplay of the sliding of branch points, entanglement release, and network deformation. The interpretation of the fast mode is on the contrary more direct and the fact that this mode really is concentration independent is confirmed by IR measurements [43].

At this stage it is interesting to combine the information from the two modes in order to get an idea of how the network looks and to check whether the information is mutually consistent. The interpretation of the fast mode suggests that a considerable amount of the surfactant molecules $(>10 \%)$ are present in the branch points. The slow mode, however, indicates the formation of a connected network already at rather small volume fractions $\left(<0.5 \%\right.$ at $\left.w_{0}=3\right)$, which could be taken as an indication that the branch points are quite far apart. For branching to have a considerable impact on the dynamics the distance between the branch points should be at most the network correlation length at the overlap volume fraction, which can be estimated from static light scattering experiments to be $50 \mathrm{~nm}$ [3]. One way to estimate the average length between branch points at higher $w_{0}$ is to take the correlation length at the volume fraction at which phase separation occurs $\left(\phi \approx 5 \%, w_{0}=4.5\right)$, since theory predicts that the number of branch points at this concentration is about 1 per blob [6]. At this volume fraction a correlation length of approximately $10 \mathrm{~nm}$ was found by static light scattering [3]. These estimates for the strand length are 
realistic as is confirmed by cryoelectron micrographs from other connected network systems, which show a strand length between 10 and $100 \mathrm{~nm}[9,10,11]$.

To estimate the amount of surfactant molecules in the branch points we still need to know the size of a branch point. Recent results by Blokhuis show that this size depends on the bending elasticity $\kappa$ and the surface tension $\sigma$ as $(\kappa / \sigma)^{1 / 2}[55]$. For a typical surfactant this would give a size in the order of $1 \mathrm{~nm}$. This could be very well somewhat higher if one takes into account that the bending elasticity of lipids can be rather high and the fact that the surface tension might be higher due to the hydration of the surfactant head groups. This also seems realistic since the micellar diameter is about $6 \mathrm{~nm}$ and it would mean that at $w_{0}=3$ about $10 \%$ of the surfactants is in the branch points and at $w_{0}=4.5$ about $50 \%$. These values seem to be in reasonable agreement with the fast mode.

\section{CONCLUDING REMARKS}

The dielectric spectroscopy data presented here are in clear disagreement with the living polymer model. This does not mean that the living polymer model, in which the mean length is a power law of the concentration, does not apply to this system. It merely shows that there are other factors like clustering and coalescence that make it very difficult to study the influence of micellar growth on the concentration independently. In order to disentangle these phenomena one would need data from as many techniques as possible.

\section{ACKNOWLEDGMENTS}

The helpful discussions with T. Odijk, E. Blokhuis, and J. Groenewold are gratefully acknowledged. We thank F. J. M. Pleijte for providing the computer programs for the analysis of the dielectric spectra.
[1] For a review: M. E. Cates and S. J. Candau, J. Phys.: Condens. Matter 2, 6869 (1990).

[2] P.-G. de Gennes, Scaling Concepts in Polymer Physics (Cornell University Press, Ithaca, NY, 1979).

[3] P. Schurtenberger and C. Cavaco, Langmuir 10, 100 (1994).

[4] For a more recent review see: M. E. Cates, in Fundamental Problems in Statistical Mechanics VII, edited by $\mathrm{H}$. van Bijeren and M. H. Ernst (Elsevier Science BV, Amsterdam, 1994).

[5] P. Schurtenberger, R. Scartazzini, L. J. Magid, M. E. Leser, and P. L. Luisi, J. Phys. Chem. 94, 3695 (1990).

[6] T. J. Drye and M. E. Cates, J. Chem. Phys. 96, 1367 (1992).

[7] F. Lequeux, Europhys. Lett. 19, 675 (1992).

[8] T. Shikata, H. Hirata, and T. Kotaka, Langmuir 5, 398 (1989).

[9] I. Harwigsson, O. Söderman, and O. Regev, Langmuir 10, 4731 (1994).

[10] D. Danido, Y. Talman, H. Levi, G. Beinert, and R. Zana, Science 269, 1420 (1995).

[11] Z. Lin, Langmuir 12, 1729 (1996).

[12] Y. Talmon, Ber. Bunsenges. Phys. Chem. 100, 364 (1996).

[13] P. A. Cirkel, D. D. P. W. Stam, and G. J. M. Koper, Coll. Surf. A (to be published).

[14] For a review see C. Boned and J. Peyrelasse, J. Surf. Sci. Technol. 7, 1 (1991).

[15] K. Shinoda, M. Araki, A. Sadaghiani, A. Khan, and B. Lindman, J. Phys. Chem. 95, 989 (1991).

[16] C. J. F. Bötcher and P. Bordewijk, Theory of Electric Polarization, 2nd ed. (Elsevier Scientific, Amsterdam, 1978), Vol. II.

[17] P. A. Cirkel, J. P. M. van der Ploeg, and G. J. M. Koper, Physica A 235, 269 (1997).

[18] A. Ott, W. Urbach, D. Langevin, P. Schurtenberger, R. Scartazzini, and P. L. Luisi, J. Phys.: Condens. Matter 2, 5907 (1990).

[19] G. J. M. Koper, C. Cavaco, and P. Schurtenberger, in 25 Years of Non-equilibrium Statistical Mechanics, edited by J. J. Brey, J. Marro, J. M. Rubi, and M. San Mighuel (Springer-Verlag, Berlin, 1995).

[20] B. H. Zimm, J. Chem. Phys. 24, 269 (1956).
[21] W. H. Stockmayer, Pure Appl. Chem. 15, 539 (1967).

[22] T. Yoshizaki and H. Yamakawa, J. Chem. Phys. 81, 982 (1984).

[23] M. Muthumakar and K. F. Freed, Macromolecules 11, 843 (1978).

[24] M. Muthumakar, Macromolecules 17, 971 (1984).

[25] M. Daoud and G. Jannink, J. Phys. I 37, 973 (1976).

[26] S. S. Patel and K. M. Takahashi, Macromolecules 25, 4382 (1992).

[27] O. Urukawa, K. Adachi, and T. Kotaka, Macromolecules 26, 2042 (1993).

[28] O. Urukawa, K. Adachi, T. Kotaka, Y. Takemoto, and H. Yashuda, Macromolecules 27, 7410 (1994).

[29] B. Baysal and W. H. Stockmayer, J. Mol. Liq. 56, 175 (1993).

[30] J. N. Israelachvili, D. J. Mitchell, and B. W. Ninham, J. Chem. Soc., Faraday Trans. 72, 1525 (1976).

[31] T. Odijk, Biophys. Chem. 41, 23 (1991).

[32] W. E. McMullen, W. M. Gelbart, and A. Ben-Shaul, J. Phys. Chem. 88, 6649 (1984).

[33] P. A. Cirkel and G. J. M. Koper (unpublished).

[34] G. Jerke, J. S. Pedersen, S. U. Egelhaaf, and P. Schurtenberger, Phys. Rev. E 56, 5772 (1997).

[35] X.-I. Wu, C. Yeung, M. W. Kim, J. S. Huang, and D. OuYang, Phys. Rev. Lett. 68, 1426 (1992).

[36] T. Kato, K. Masa-aki, and T. Seimiya, Langmuir 11, 1867 (1995).

[37] P. van der Schoot, Europhys. Lett. 39, 25 (1997), and references therein.

[38] P. van der Schoot and M. E. Cates, J. Chem. Phys. 101, 5040 (1994).

[39] T. J. Drye and M. E. Cates, J. Chem. Phys. 98, 9790 (1993).

[40] C. Cavaco, Ph.D. thesis, ETH Zürich, 1994.

[41] U. Kaatze, R. Henze, and H. Eibl, Biophys. Chem. 10, 351 (1979).

[42] R. Pottel, U. Kaatze, and St. Müller, Ber. Bunsenges. Phys. Chem. 82, 1086 (1978).

[43] P. A. Cirkel, J. P. M. van der Ploeg, and G. J. M. Koper, Prog. Colloid Polym. Sci. 105, 204 (1997).

[44] D. Capitani, A. L. Segre, R. Sparapani, M. Giustini, R. Scar- 
tazzini, and P. L. Luisi, Langmuir 7, 250 (1991).

[45] C. Cavaco and P. Schurtenberger, Helv. Phys. Acta 67, 227 (1994).

[46] A. Khatory, F. Kern, F. Lequeux, J. Appell, G. Porte, N. Morie, A. Ott, W. Urbach, and F. Kern, Langmuir 9, 933 (1993).

[47] J. Appel, G. Porte, A. Khatory, F. Kern, and S. J. Candau, J. Phys. II 2, 1045 (1992).

[48] G. J. M. Koper, W. F. C. Sager, J. Smeets, and D. Bedeaux, J. Phys. Chem. 99, 13291 (1995).

[49] Y. Gefen, A. Aharony, and S. Alexander, Phys. Rev. Lett. 50, 77 (1983).
[50] D. Vandembroucq, A. C. Boccara, and S. Roux, Europhys. Lett. 30, 209 (1995).

[51] A. Ott, J. P. Bouchaud, D. Langevin, and W. Urbach, Phys. Rev. Lett. 65, 2201 (1990).

[52] K. Elleuch, F. Lequeux, and P. Pfeuty, J. Phys. I 5, 465 (1995).

[53] Y. Bohbot, A. Ben Shaul, R. Granek, and W. N. Gelbart, J. Chem. Phys. 103, 8764 (1995).

[54] T. Tlusty, S. A. Safran, R. Menes, and R. Strey, Phys. Rev. Lett. 78, 2616 (1997).

[55] E. Blokhuis (unpublished). 